\title{
Nature or Nurture: Nutritional Approach to Periodontal Therapy
}

\author{
Dr.Amrita ${ }^{1}$, Dr. Megha Phogat Rana ${ }^{2}$, Dr. Mahendra Pratap ${ }^{3}$ \\ ${ }^{1}$ Post Graduate, Department of Periodontology, Seema Dental College and Hospital, Rishikesh \\ ${ }^{2}$ Reader, Department of Periodontology, Seema Dental College and Hospital, Rishikesh \\ ${ }^{3}$ Post Graduate, Department of Orthodontics, Teerthankar Mahaveer Dental College and Hospital, Moradabad
}

\begin{abstract}
Periodontitis is a ubiquitous chronic inflammatory disease affecting supporting structures of the teeth. With improved understanding of the mechanism behind periodontal tissue devastation, the role of nutrients has led to an increased understanding of association between nutrition and periodontal diseases. Nutrition plays a very significant role in man's development, health and welfare, therefore nutrition has correlation to the natural resolution of the periodontal diseases. The protective defensive role of nutrients and the advent of modern genomic measurement tools have led to an increased interest that how diet affect the inflammatory mechanisms underlying severe periodontitis.
\end{abstract}

Keywords: Periodontitis, nutrition

\section{Introduction}

Periodontitis is defined as "a chronic inflammatory disease of the supporting tissues of the teeth caused by specific microorganisms or groups of specific micro-organisms, initiating progressive destruction of the periodontal ligament and alveolar bone with pocket development, recession, or both". [1] It is characterized by a dysregulation of host inflammatory/immune response to plaque bacteria in susceptible individuals, however the host response exhibits wide heterogeneity in common with other chronic inflammatory diseases, and with relatively minor shifts in host response resulting in disease development and progression in susceptible people. A variety of risk factors have been identified that modify the host response and thereby shifting the biological balance from health to disease. These factors can be characterized as genetic, environmental (e.g. stress, bacterial challenge) and lifestyle/behavioral (e.g. exercise, nutrition, smoking) ${ }^{[2]}$ It is widely regarded as the second most common disease world-wide, after dental decay and in the United States has a prevalence of $30-50 \%$ of the population, but only about $10 \%$ have severe forms. ${ }^{[3]}$

The increasing interest in the association between nutrition and periodontal disease is raising due to the improved understanding of the mechanisms behind periodontal tissue destruction, the potential protective role of nutrients and the advent of modern genomic measurement tools have led to a common dietary chemicals act on the human genome, either directly or indirectly, to alter gene expression or structure. Genes are important in determining the function, but nutrition is able to modify the degree of gene expression. ${ }^{[4]}$

\section{Evolution of Nutrigenomics}

It was on April 14, 2003 that precise birth date of the genomic era was decided and when Human Genome Projectwas ${ }^{[5]}$ launched with the participation of former U.S President Bill Clinton and former British PM Tony Blair which contained the complete sequencing of the human genome. It was the time when a new era in biological and medical science was beginning. This is often referred to as the 'omics'-revolution.

In 2004, NuGo (European Nutrigenomics Organization) was born and funded until June 2010.

In 2007, Nestle Research Center joined the industrial platform of the Kluyver Centre for Genomics of industrial fermentation, Netherlands.

In 2008, US Berkeley scientist predicted human genome tests within five years for $\$ 100 .{ }^{[6]}$

\section{Nutrition and periodontal inflammation}

Nutrition can also influence the levels of inflammation in periodontitis. Experimental gingivitis model studies have shown increased levels of bleeding on probing when participants were fed with a diet high in carbohydrates when compared to those on a low sugar diet. ${ }^{[7]}$ It was further supported by a study investigating volunteers placed on a primitive diet which was high in fiber, anti-oxidants, and fish oils, but low in refined sugars and with no oral hygiene measures. ${ }^{[8]}$

Plaque biofilminitially is considered to be the most triggering factor in periodontal disease, ${ }^{[9]}$ but most tissue destruction results from an abnormal inflammatory immune response in patients predisposed to the condition. ${ }^{[10]}$ It is the hyper- 


\section{International Journal of Science and Research (IJSR) \\ ISSN (Online): 2319-7064 \\ Index Copernicus Value (2013): 6.14 | Impact Factor (2015): 6.391}

inflammatory response which fails to eradicate the causative pathogens and generates prolonged release of neutrophil proteolytic enzymes, pro-inflammatory mediators and reactive oxygen species (ROS), which in turn destroys the periodontal attachment. ${ }^{[1]]}$ The etiology of an inflated inflammatory immune response is complex, but several common features of hyper-inflammation underlie the major chronic human diseases. ${ }^{[12]}$

The term "reactive oxygen species" (ROS) has been implicated to include molecules such as hydrogen peroxide (H2O2), hypochlorous acid (HOCI) and singlet oxygen (1O2), which are capable of radical transformation in the extra and intra-cellular environments but are not radical in nature. Most reactive oxygen species have extremely short half-lives, but they can cause substantial tissue damage by initiating free radical chain reactions. Therefore the body contains a number of protective Anti-Oxidant (AO) mechanisms, which tends to remove harmful oxidants (ROS), as soon as they form, or to repair the damage caused by reactive oxygen species in vivo. [13]

Antioxidants are defined as "those substances which when present at low concentrations, compared to an oxidisable substrate, will significantly delay or inhibit oxidation of that substrate". ${ }^{[14]}$

In patients with early onset periodontitis, low superoxide production is seen after myeloperoxidase stimulus of blood neutrophils. Even in patients with adult periodontitis, increased generation of free oxygen radicals and increased ratio between released elastase and lactoferrin is seen in peripheral neutrophils thus causing tissue destruction. ${ }^{[11]}$

Enhanced ROS generation by peripheral neutrophils in patients with both chronic and aggressive diseaseget stimulated with opsonized bacteria associated with periodontal disease (Fusobacterium nucleatum, Actinobacillus actinomycetemcomitans). This is suggestive of hyper-active phenotype of peripheral neutrophils that may have local tissue damaging consequences. ${ }^{[15]}$

Evidence for the presence and role of reactive oxygen species in periodontal tissue damage:

Halliwell proposed four criteria, similar to that proposed by Robert Koch in 1884, to establish causal relationship between an organism and disease. ${ }^{[11]}$

1) Reactive Oxygen Species or the oxidative damage caused must be present at the site of injury.

2) The time course ofReactive Oxygen Species formation or the oxidative damage caused should occur before or at the same time as tissue injury.

3) Direct application of Reactive Oxygen Species over a relevant time course to tissues at concentrations found in vivo should reproduce damage similar to that observed in the diseased tissue.
4) Removing or inhibitingReactive Oxygen Species formation should decrease tissue damage to an extent related to their anti-oxidant action in vivo.

The presence of macronutrients and micronutrients modulatepro-inflammatory and anti-inflammatory cascades, which influences a person's baseline inflammatory status. The functionality of nutrients in human biology is to act as molecular signals which are capable of modulating gene and protein expression at a molecular level that extends beyond that of being just fuels for energy production and co-factors in the metabolism. ${ }^{[16]}$

Diets high in complex carbohydrates are generally healthy, whereas those rich in refined carbohydrates can be major causes of chronic inflammation. ${ }^{[16,17]}$ Elevated glucoseand lipid levels generate Reactive Oxygen Species at a rate that exceeds endogenous anti-oxidant defenses, and leads to oxidative stress. The "post-prandial dys-metabolism" plays a role in the genesis of inflammation.Multiple elevation in glucose eventually leads to chronic inflammatory pathologies. ${ }^{[17,18,19]}$ Diet-induced hyperlipidemia induces oxidative stress and down regulates the inflammation, ${ }^{[20]}$ and lipoproteins formed by liver hepatocytes can be converted to free fatty acids within the circulation and taken up by adipocytes, thus acting as a basis of pro-inflammatory adipokines.

In the states of oxidative stress, lipid peroxidation (a chain reaction induced by ReactiveOxygen Species attack on the polyunsaturated fatty acid [PUFA] side-chains of lipid membranes) arises, ${ }^{[11]}$ lowdensity lipoproteins are oxidized and bind to "toll-like receptors" (TLR-2/4) that are a group of pattern recognition receptors on inflammatory cell membranes, triggering nuclear factor-каppa $\mathrm{B}$ activation via the protein-kinase-C enzyme and other related pathways. NF$\kappa \mathrm{B}$ transcribes several pro-inflammatory cytokines. ${ }^{[20]}$

An inverse relationship between reduced concentrations of plasma total anti-oxidants and vitamin $\mathrm{C}$ are seen that in turn increases the prevalence of periodontitis as the anti-oxidant depletion in periodontitis occurs locally in the periodontium ${ }^{[21]}$ and within plasma. ${ }^{[22]}$ Intervention studies involving patients with periodontitis and demonstrable vitamin deficiencies, however, are scarce.

Therefore, the basis for individual vitamin supplements as therapeutics in the absence of frank deficiency is flawed because of the potentialfor in vivo vitamin-radical formation; the need for co-operative anti-oxidant cascades to be augmented and the need for only subtle increases in antioxidant status is necessary to down regulate pro inflammatory gene transcription. ${ }^{[11]}$

\section{Nutrition and Gene Expression}

Nutrigenomics is an emerging field of science and technology unrevealing inter-relationships between nutrients and human genome using modern tools such as transcriptomics, 


\section{International Journal of Science and Research (IJSR) \\ ISSN (Online): 2319-7064 \\ Index Copernicus Value (2013): 6.14 | Impact Factor (2015): 6.391}

metabolomics, epigenomics and proteomics. It implies that nutrition and genetics both play a significant role in the maintenance of human health as well as the development of lethal diseases. ${ }^{[23]}$ It aims to reveal the relationship between nutrition and the genome and to provide the scientific basis for improved public health through dietary means.

The term genomics describes the process by which all genes present in the genome of a given species can be mapped, sequenced and characterized. Extensions of genomics, such as structural and / or comparative genomics, are also being used to characterize the genome in greater detail.

Transcriptomics is the term used to describe the approach in which mRNA, and consequently gene expression, is analyzed in a biological sample under certain conditions at a given point in time.

Proteomics take this analysis further and aims to characterize all proteins in a biological sample at the functional level.

Metabolomics is used to describe quantitative analysis of all metabolites in a biological system such as cell, tissue or biological fluid (blood, plasma and saliva). ${ }^{[24]}$

Nutrigenomics shows a new way of working with nutrition and now, the knowledge of how food impedes with the genetic code and how the organism responds to these interferences and with the phenotype can be explained. ${ }^{[24]}$

Nutrigenomics is both the examination of how nutrients affect genes (i.e. influence gene expression and function) and how genes affect diet (i.e. what an individual eats and how an individual responds to nutrients), with the latter sometimes being referred to as Nutrigenetics. Nutrigenomics can include the full spectrum of research strategies from basic cellular and molecular biology to clinical trials, epidemiology and population health. These different experimental approaches can be used to improve our understanding of how nutrition affects various health outcomes, and current trends in personalized nutrition have focused on the role of genetic variation to understand why some individuals respond differently from others to the same nutrients consumed. ${ }^{[25]}$ Practical Applications of Nutrigenomics ${ }^{[26]}$

1) Genes and proteins expressed differentially in health and disease that are modifiable by nutrients are identified.

2) Genes, proteins, and metabolites are influenced by specific nutrients that are known to be beneficial or harmful are identified.

3) To identify genes, proteins, and metabolites that are altered by dietary fats associated with cardiovascular disease.

4) To identify genes, proteins, and metabolites that is altered by omega 3 fatty acids.

5) Genetic variations that alter the nutrient-gene interactions in applications 1 and 2 are identified.

\section{Nutrigenomics and Periodontics}

It has been implicated that nutritional plays a role in the regulation of periodontal disease that can be attributed to the presence of reactive oxygen species. The studies performed in the 1980s (reviewed by Chapple and Matthews) using individual vitamin supplements investigated only patients who did not have vitamin deficiency, who were in worthy periodontal health or both.

A latest study ofpatients with metabolic syndrome provided early indications of the potential of anti-oxidants found naturally in foods to reduce periodontal inflammation(at clinical and biomarker levels) in patients with disease. ${ }^{[27]}$ Additionally, researchers have proposed reduced glutathione (GSH), the key intra-cellular anti-oxidant redox-regulator of $\mathrm{NF}-\kappa \mathrm{B}$, as a novel approach to downregulation of hyperinflammatory events. ${ }^{[28]} \mathrm{GSH}$ levels appear depleted in periodontitis ${ }^{[29]}$ and methods of enhancing intracellular GSH may prove beneficial.

PUFAs of the omega-3 form ( $\omega$-3PUFAs) found in fish oils lower postprandial triglyceride levels ${ }^{[30]}$ and confer antiinflammatory and cardiovascular protective effects. ${ }^{[31]} \omega$ 3PUFAs also inhibit lipid mediators of inflammation (such as prostaglandin E2, arachidonic acid, 5-lipoxygenase and cyclooxygenase), modulate lymphokine production and upsurge antioxidant capacity, ${ }^{[32-34]}$ and are reported to decrease osteoclast activity. ${ }^{[35]}$ Kesavalu and colleagues ${ }^{[36]}$ demonstrated that rats infected with Porphyromonasgingivalis and fed with a diet rich in $\omega-3$ PUFAs for 22 weeks experienced less bone loss than did control rats fed a diet rich in n-6 PUFAs. Gene expression echelons of IL-1 $\beta$ and TNF $\alpha$ declined, and those of interferon- $\gamma$ and intracellular antioxidant enzymes increased. ${ }^{[37]}$ One mode of action of PUFAs include the downregulation of pro-inflammatory gene expression via the nuclear peroxisome proliferator activated receptors (PPARs) and others include inflammation resolving mediators derived from $\omega-3$ PUFAs (resolvins). ${ }^{[38]}$

Thus nutrigenomic studies have highlighted theimportance of variations in gene structure (forinstance, at the transcription factor bindingsite) on differential responses of patients to specificnutrients.

Di Silvestro RA et al conducted a study in 2009 and showed that one minute rinsing with a mouthwash containing pomegranate extract successfully reduced the amount of micro-organisms cultured from dental plaque. ${ }^{[39]}$

The effect of Morinda citrifolia L. fruit juice significantly mitigated the gingival inflammation. The combination of good oral hygiene and administration of this juice was a promising treatment for gingivitis and periodontitis because of its strong anti-inflammatory effects.

Merchant et al in 2006 conducted a prospective, observational study that was carried out over 14 years and revealed that men

Volume 5 Issue 5, May 2016 www.ijsr.net 


\section{International Journal of Science and Research (IJSR) \\ ISSN (Online): 2319-7064 \\ Index Copernicus Value (2013): 6.14 | Impact Factor (2015): 6.391}

with high consumption of wholegrain were $23 \%$ less likely to develop periodontitis. ${ }^{[40]}$

A randomized double blind clinical trial conducted by Vander Veldon in 2010 investigated the potential clinical benefits of a powdered fruit and vegetable juice concentrate on the treatment of patients with chronic periodontitis showed there was an increased pocket depth reduction following standard non-surgical therapy compared to a placebo group. ${ }^{[41]}$

In 1984 Ashley in his research studies using an experimental gingivitis model showed increased levels of bleeding on probing when participants were fed with a diet high in carbohydrates when compared to those on a low sugar diet.

${ }^{[42]}$ This finding was supported further by Robbinson in 2000 in his study investigating volunteers placed on a primitive diet which was high in fiber, anti-oxidants, and fish oils, but low in refined sugars and with no oral hygiene measures. ${ }^{[8]}$

\section{Nutritional approach to periodontal therapy}

Periodontitis is associated with/low serum/plasma micronutrient levels that may result from dietary and/or life-style factors as well as nutri-genetic characteristics. Primary evidence suggests beneficial results from nutritional interventions; supporting the contention that daily intake of certain nutrients should be at the higher end of recommended daily allowances. For inhibition and management of periodontitis daily nutrition should include sufficient antioxidants, vitamin D, and calcium. Insufficient anti-oxidant levels may be managed by higher intake of vegetables, berries, and fruits (e.g. kiwi fruit), or by phytonutrient supplementation. Anti-oxidant micronutrient deficiencies can be met by a higher intake of vegetables, fruits, and berries. ${ }^{[43]}$ The majority of patients are not anti-oxidant vitamin deficient and monovitamin supplements may be associated with toxicity effects. Current evidence shows some benefit in reducing gingival inflammation from vitamin $\mathrm{C}$ supplementation. The most appropriate sources of vitamin $\mathrm{C}$ are natural fruits such as kiwi fruit. ${ }^{[44]}$ The most appropriate sources of polyphenolicflavonoids and carotenoids are from natural fruit/vegetable/berry intake or the use of whole fruit, vegetable, and berry concentrates. An initial intervention study with a powdered capsular form of the latter phytonutrients showed promise as an adjunctive approach to standard periodontal therapy in improving pocket depth reductions. ${ }^{[45]}$

The seeds of Garcinia mangostana are reported to contain vitamin C. A composition in the form of a bio-degradable gel, chip or ointment is provided for the treatment of periodontitis, comprising an anti-microbial or anti-bacterial activity against periodontal pathogen and forms a liquid crystal structure on contacting with gingival fluid, which releases active ingredients gradually, to provide a sustained release dosage form.

The observed differences in the response of an individual to dietary modification can be attributed to differences in their genetic make-up, which emphasizes the importance of exploring the role of nutrient-gene interactions in the development of chronic diseases. Oral health scientists now have the opportunity to study nutrient-gene interactions and how diet affects the inflammatory mechanisms underlying severe periodontitis.

\section{Dietary recommendations for periodontal disease management}

The recommendations of the 2011 European Workshop on Periodontology ${ }^{[22]}$ suggest that the dental team should consider including advice to all patients on increasing levels of fish oils, fiber, fruits and vegetables and to reduce levels of refined sugars as part of a periodontal prevention/treatment regime and a general health benefit message.

\section{Conclusion}

In this post-genomic era, the association between the potential role of nutrients and periodontal diseases is well established. It is known that the interaction between the genotype and diet are important in determining periodontal disease risk.

\section{References}

[1] Newman MG, Takei HH, Klokkevold PR, Carranza FA. Carranza's Clinical Periodontology, 10th Edition, Elsevier 2006: p103-4.

[2] Van Dyke TE. Pro-resolving lipid mediators: potential for prevention and treatment of periodontitis. J Clin Periodontol 2011; 38 (suppl 11):119-25.

[3] White DA, Tsakos G, Pitts NB, Fuller E, Douglas GV, Murray JJ. Adult dental health survey 2009: Common oral health conditions and their impact on the population. Br Dent J 2012; 212(11):567-72.

[4] Shivanand S, S Savita, K Rithesh, Singh N. Nutrigenomics: a new paradigm for revealing periodontal inter-relationships. Journal of Biomedical and Pharmaceutical Research 5;(1):07-15.

[5] Garver KL, Garver B. The human genome project and eugenic concerns. Am J Hum Genet 1994; 54(1):148-58.

[6] Priyadharshini RS, Vijayalakshmi R, Ambalavanan N, Ramakrishnan T, Logaranjani A. Nutrigenomics in periodontics- an overview. Int J Cur Res Rev 2016; 8(1):31-5.

[7] Sidi A, Ashley F. Influence of frequent sugar intakes on experimental gingivitis. J Periodontol 1984; 55:419-23.

[8] Baumgartner S, Imfeld T, Schicht. The impact of the Stone Age diet on gingival conditions in the absence of oral hygiene. J Periodontol2009; 80:759-68.

[9] Axelsson P, Albandar JM, Rams TE. Prevention and control of periodontal diseases in developing and industrialized nations. Periodontol 2000 2002; (29):23546.

[10] Page RC, Kornman KS. The pathogenesis of human periodontitis: an introduction. Periodontol 2000 1997; 14:9-11.

Volume 5 Issue 5, May 2016 


\section{International Journal of Science and Research (IJSR) \\ ISSN (Online): 2319-7064 \\ Index Copernicus Value (2013): 6.14 | Impact Factor (2015): 6.391}

[11] Chapple ILC, Matthews JB. The role of reactive oxygen and anti-oxidant species in periodontal tissue destruction. Periodontol 2000 2007; 43(1):160-232.

[12] Libby P, Ridker PM, Maseri A. Inflammation and atherosclerosis. Circulation 2002 105; (9):1135-43.

[13] Waddington R, Moseley R, Embery G. Periodontal disease mechanisms: Reactive oxygen species: a potential role in the pathogenesis of periodontal diseases. Oral Diseases 2000; 6(3):138-51.

[14] Wanasundara P, Shahidi F. Antioxidants: Science, technology, and applications: John Wiley and Sons, Inc; 2005.

http://onlinelibrary.wiley.com/doi/10.1002/047167849X. bio002/full.

[15] Whyte G, Seymour GJ, Cheung K, Robinson MF. Chemiluminescence of peripheral polymorphonuclear leukocytes from adult periodontitis patients. J Clin Periodontol 1989; 16(2):69-74.

[16] Keefe JH, Gheewala NM, Keefe JO. Dietary strategies for improving post-prandial glucose, lipids, inflammation, and cardio-vascular health. J Am Coll Cardiol 2008; 51(3):249-55.

[17] Mitrou PN, Kipnis V, Thiebaut AC. Mediterranean dietary pattern and prediction of all-cause mortality in a US population: results from the NIH-AARP diet and health study. Arch Int Med 2007; 167(22):2461-68.

[18] Keefe J, Bell D. Post-prandial hyperglycemia/hyperlipidemia (postprandial dysmetabolism) is a cardiovascular risk factor. Am J Cardiol 2007;100(5):899-904.

[19] Weissman A, Lowenstein L, Peleg A, Thaler I, Zimmer EZ. Power spectral analysis of heart rate variability during the $100 \mathrm{~g}$ oral glucose tolerance test in pregnant women. Diabetes Care 2006; 29(3):571-74.

[20] Chapple ILC. Potential mechanisms underpinning the nutritional modulation of periodontal inflammation. J Am Dent Assoc 2009; 140:178-84.

[21] Chapple IL, Brock GR, Milward MR, Ling N, Matthews JB. Compromised GCF total anti-oxidant capacity in periodontitis: cause or effect? J ClinPeriodontol 2007; 42(2):103-10.

[22] Chapple IL, Milward M, Dietrich T. The prevalence of inflammatory periodontitis is negatively associated with serum anti-oxidant concentrations. J Nutr 2007; 137(3):657 64.

[23] Siddique R, Iftikhar H, Ejaz R, Karim T. NutrigenomicsAn emerging field. National University of Sciences and Technology 2013; 1-25.

[24] Dang TS, Walker M, Ford D, Valentine RA. Nutrigenomics: the role of nutrients in gene expression. Periodontol 2000 2014; 64:154-60.

[25] IUFOST Scientific Information Bulletin (SIB) March 2012.

[26] Kornman KS, Martha PM, Duff GW. Genetic variations and inflammation: a practical nutrigenomics opportunity. Nutrition 2004 20; (1):44-9.

[27] Jenzsch A, Eick S, Rassoul F, Purschwitz R, Jentsch H. Nutritional intervention in patients with periodontal disease: clinical, immunological and microbiological variables during 12 months. Br J Nutrition 2008; 20:1-7.

[28] Chapple IL. Reactive oxygen species and anti-oxidants in inflammatory diseases. J ClinPeriodontol 1997; 24(5):287-96.

[29] Chapple IL, Brock G, Eftimiadi C, Matthews JB. Glutathione in gingival crevicular fluid and its relation to local anti-oxidant capacity in periodontal health and disease. Mol Pathol 2002; 55(6):367-73.

[30] Park Y, Harris W. Omega-3 fatty acid supplementation accelerates chylomicron triglyceride clearance. J Lipid Res 2003; 44 (3):455-63.

[31] Yokoyama M, Origasa H, Matsuzaki M, et al. Effects of eicosopentaenoic acid on major coronary events in hypercholesterolaemic patients (JELIS): a randomized open-label, blinded end-point analysis. Lancet 2007 369; (9567):1090-98.

[32] Alam SQ, Bergens BM, Alam BS. Arachidonic acid, prostaglandin E2 and leukotriene $\mathrm{C} 4$ levels in gingival and sub-mandibular salivary glands of rats fed diets containing n-3 fatty acids. Lipids 1991 26(11):895-900.

[33] Blok WL, Vogels MT, Curfs JH, Eling WM, Buurman WA, van der Meer JW. Dietary fish-oil supplementation in experimental gram-negative infection and in cerebral malaria in mice. J Infect Dis 1992; 165(5):898-903.

[34] Fernandes G, Venkataraman J. Role of omega-3 fatty acids in health and disease. Nutr Res 1993; 13:S19-45.

[35] Campan P, Planchand PO, Duran D. Pilot study of n-3 polyunsaturated fatty acids in the treatment of human experimental gingivitis. J Clin Periodontol 1997; 24(12):901-13.

[36] Kesavalu L, Vasudevan B, Raghu B, et al. Omega-3 fatty acid effect on alveolar bone loss in rats. J Dent Res 2006; 85(7):648-52.

[37] Kesavalu L, Bakthavatchalu V, Rahman MM. Omega-3 fatty acid regulates inflammatory cytokine/mediator messenger RNA expression in Porphyromonas gingivalis induced experimental periodontal disease. Oral Microbiol Immunol 2007; 22(4):232-39.

[38] Serhan CN. Controlling the resolution of acute inflammation: a new genus of dual anti-inflammatory and pro-resolving mediators. J Periodontol 2008; 79(8 suppl):1520-26.

[39] Di Silvestro RA, Di Silvestro DJ. Pomegranate extract mouth rinsing effects on saliva measures relevant to gingivitis risk. Phytother Res 2009; 23(8):1123-27.

[40] Merchant AT, Pitiphat W, Franz M, Joshipura KJ. Wholegrain and fiber intakes and periodontitis risk in men. Am J Clin Nutr 2006; 83(6):1395-1400.

[41] Semba RD, Tang AM. Micro-nutrients and the pathogenesis of human immune-deficiency virus infection. Br J Nutr 1999; 81(3):181-89.

[42] Sidi AD, Ashley FP. Influence of frequent sugar intakes on experimental gingivitis. J Periodontol 1984; 55(7):419-23.

[43] Van der Velden U, Kuzmanova D, Chapple ILC. Micronutritional approaches to periodontal therapy. J Clin Periodontol 2011; 38 (Suppl. 11):142-58. 


\section{International Journal of Science and Research (IJSR) \\ ISSN (Online): 2319-7064}

Index Copernicus Value (2013): 6.14 | Impact Factor (2015): 6.391

[44] Burrill D. Relationship of blood plasma vitamin C level to gingival and periodontal disease. J Dent Res 1942; 21:353-63.

[45] Phillips CM. Nutrigenetics and metabolic disease: Current status and implications for personalized nutrition. Nutrients 2013; 5:32-57.

\section{Author Profile}

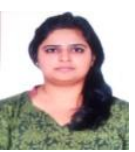

Dr. Amrita is a post-graduate student in the Department of Periodontology, Seema Dental College and Hospital, Rishikesh. Graduated in 2011 from S.J.M Dental College and Hospital, Chitradurga, Karnataka.

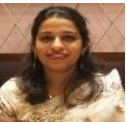

Dr. Megha Phogat Rana is Reader in the Department of Periodontology, Seema Dental College and Hospital, Rishikesh. Graduated in 2006 from DAVYamunanagar and completed post-graduation in 2010 from BBD College of Dental Sciences, Lucknow.

Dr. Mahendra Pratapis a post graduate student in the Department of Orthodontics, Teerthanker Mahaveer Dental College \& Hospital, Moradabad. Graduated in 2012 from Teerthanker Mahaveer Dental College \& Hospital, Moradabad.

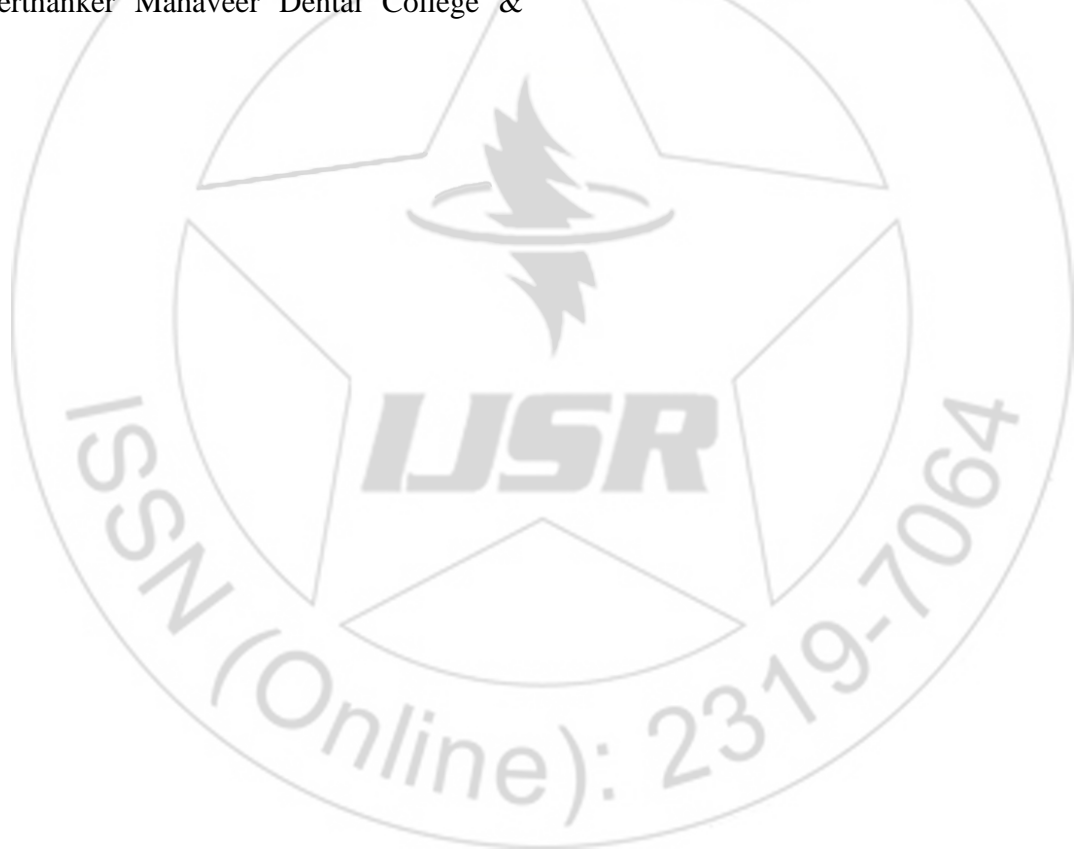

Volume 5 Issue 5, May 2016 www.ijsr.net 